Applied Mathematical Sciences, Vol. 8, 2014, no. 166, 8295 - 8307

HIKARI Ltd, www.m-hikari.com

http://dx.doi.org/10.12988/ams.2014.411917

\title{
Hitting Probabilities for Random Convex Bodies and Lattices of Triangles
}

\author{
Uwe Bäsel \\ HTWK Leipzig, \\ Fakultät Maschinenbau und Energietechnik, \\ 04251 Leipzig, Germany
}

Dedicated to Professor Marius Stoka on the occasion of his $80^{\text {th }}$ birthday

Copyright (C) 2014 Uwe Bäsel. This is an open access article distributed under the Creative Commons Attribution License, which permits unrestricted use, distribution, and reproduction in any medium, provided the original work is properly cited.

\begin{abstract}
In the first part of this paper, we obtain symmetric formulae for the probabilities that a plane convex body hits exactly $1,2,3,4,5$ or 6 triangles of a lattice of congruent triangles in the plane. Furthermore, a very simple formula for the expectation of the number of hit triangles is derived.
\end{abstract}

Mathematics Subject Classification: 60D05, 52A22

Keywords: Random convex sets, hitting probabilities, intersection probabilities, lattice of triangles, Buffon's problem, Buffon's needle problem

\section{Introduction}

We consider the random throw of a planar convex body $\mathcal{C}$ onto a plane with an unbounded lattice $\mathcal{R}_{a, b, c}$ of congruent triangles with sides $a, b, c$, and opposite angles $\alpha, \beta, \gamma$, respectively. (An example is shown in Fig. 1.) As usual, we denote the altitudes from the sides $a, b, c$ respectively as $h_{a}, h_{b}, h_{c}$, and have

$$
h_{a}=b \sin \gamma=c \sin \beta, \quad h_{b}=a \sin \gamma=c \sin \alpha, \quad h_{c}=a \sin \beta=b \sin \alpha .
$$


$\mathcal{R}_{a, b, c}$ is the union of three families $\mathcal{R}_{h_{a}}, \mathcal{R}_{h_{b}}$ and $\mathcal{R}_{h_{c}}$ of parallel lines,

$$
\begin{aligned}
& \mathcal{R}_{h_{a}}=\left\{(x, y) \in \mathbb{R}^{2} \mid x \sin \beta+y \cos \beta=k h_{a}, k \in \mathbb{Z}\right\}, \\
& \mathcal{R}_{h_{b}}=\left\{(x, y) \in \mathbb{R}^{2} \mid x \sin \alpha-y \cos \alpha=k h_{b}, k \in \mathbb{Z}\right\}, \\
& \mathcal{R}_{h_{c}}=\left\{(x, y) \in \mathbb{R}^{2} \mid y=k h_{c}, k \in \mathbb{Z}\right\} .
\end{aligned}
$$

Every triangle of $\mathcal{R}_{a, b, c}$ is congruent to the triangle

$$
\mathcal{T}:=\left\{(x, y) \in \mathbb{R}^{2} \mid 0 \leq y \leq h_{c}, y \cot \alpha \leq x \leq c-y \cot \beta\right\}
$$

Two triangles form a parallelogram that is congruent to the parallelogram

$$
\mathcal{Q}:=\left\{(x, y) \in \mathbb{R}^{2} \mid 0 \leq y \leq h_{c}, y \cot \alpha \leq x \leq c+y \cot \alpha\right\},
$$

see Fig. 1 and 4. The area $Q$ of $\mathcal{Q}$ is given by

$$
Q=a b \sin \gamma=a c \sin \beta=b c \sin \alpha .
$$

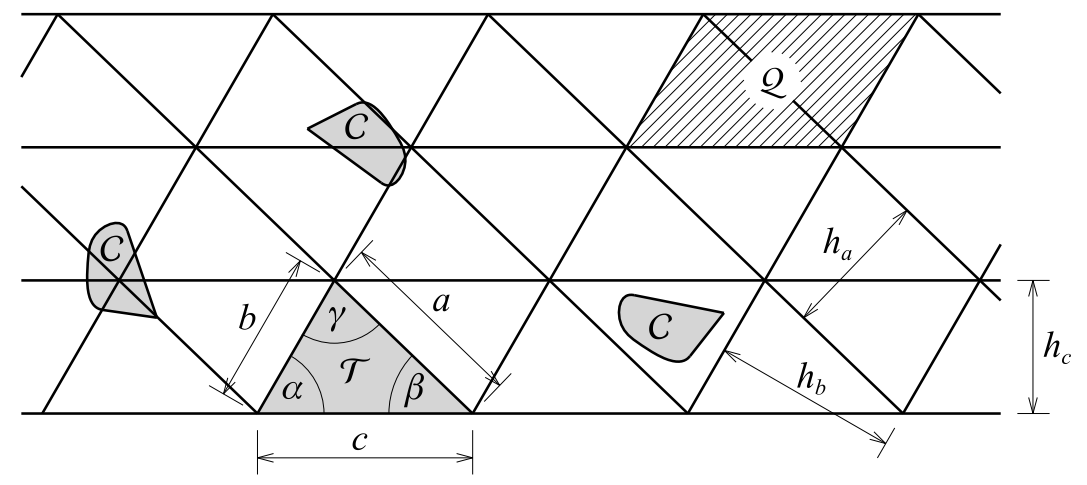

Fig. 1: Lattice $\mathcal{R}_{a, b, c}$ and convex body $\mathcal{C}$

Let $\mathcal{C}$ be provided with a fixed reference point $O$ inside $\mathcal{C}$ and a fixed oriented line segment $\sigma$ starting in $O$ (see Fig. 4), and let $\phi$ be the angle between a fixed direction in the plane and segment $\sigma$. The random throw of $\mathcal{C}$ onto $\mathcal{R}_{a, b, c}$ is defined as follows: The coordinates $x, y$ of $O$ are random variables uniformly distributed in $\left[y \cot \alpha, h_{b} \csc \alpha+y \cot \alpha\right]$ and $\left[0, h_{c}\right]$, respectively; the angle $\phi$ is a random variable uniformly distributed in $[0,2 \pi]$. All three random variables are stochastically independent. Let $u$ denote the perimeter of $\mathcal{C}, F$ the area of $\mathcal{C}$, and $s: \mathbb{R} \rightarrow \mathbb{R}, \phi \mapsto s(\phi)$ the support function of $\mathcal{C}$ with reference to the point $O$. We use $s$ in the following sense (see Fig. 2): $\mathcal{C}$ rotates about the origin of a Cartesian $x, y$-coordinate system with rotation angle $\phi$ between the $x$-axis and segment $\sigma$, whereby $O$ coincides with the origin. The support line, touching $\mathcal{C}$, is perpendicular to the $x$-axis. Now, the support function $s$ is the distance between the origin and the support line. In [11, pp. 2-3], the support 
function, say $\tilde{s}$, is defined for fixed $\mathcal{C}$ and moving support line. Clearly, the relation between these two definitions is given by $s(\phi)=\tilde{s}(-\phi)$ if the fixed body $\mathcal{C}$ is in the position with $\phi=0$, and $O$ coinciding with the origin. The width of $\mathcal{C}$ in the direction $\phi$ is given by $w: \mathbb{R} \rightarrow \mathbb{R}, \phi \mapsto w(\phi)=s(\phi)+s(\phi+\pi)$.

Markov [6, pp. 169-173] calculated the probability $p(1)$ that a line segment (needle) of length $\ell$ hits exactly one triangle of $\mathcal{R}_{a, b, c}$. He found

$$
p(1)=1+\frac{\ell^{2}\left(\alpha a^{2}+\beta b^{2}+\gamma c^{2}\right)}{2 \pi Q^{2}}-\frac{\ell(4 a+4 b+4 c-3 \ell)}{2 \pi Q} .
$$

Pettineo [7, pp. 289-294] and Böttcher [3, pp. 3-10] calculated the probability $p$ that the needle hits at least one line of $\mathcal{R}_{a, b, c}$. Clearly, $p=1-p(1)$. Although giving equal results, the formulae of Markov, Pettineo, and Böttcher are quite different. Santaló [10, pp. 167-170] (see also [11, pp. 140-141]) calculated the probabilities that the needle hits $0,1,2$ or 3 lines of the lattice $\mathcal{R}_{a, a, a}$ of equilateral triangles. Duma and Stoka [4, pp. 16-20] obtained the probabilities that $\mathcal{C}$ hits at least one line, and exactly three lines of $\mathcal{R}_{a, b, c}$. Ren and Zhang [8, pp. 320-321] (see also [9, pp. 72-73]) computed the probabilities for the number of hit lines for a bounded convex body $\mathcal{C}$ with no size restriction, and a lattice of parallelograms. Bäsel and Duma [1], [2] calculated the probabilities that $\mathcal{C}$ hits exactly 1, 2, 3 or 4 parallelograms for two kinds of parallelogram lattices.

\section{Hitting probabilities}

In the following, we choose $\phi$ as the angle between the direction perpendicular to the sides $b$ and segment $\sigma$ (see Fig. 4). We assume that for every angle $\phi$, $0 \leq \phi<2 \pi$, there is a position of the reference point $O$ so that $\mathcal{C}$ with angle $\phi$, denoted as $\mathcal{C}_{\phi}$, is entirely contained in exactly one triangle $\mathcal{T}$ of $\mathcal{R}_{a, b, c}$. Therefore, we consider the triangle $\mathcal{T}^{*}(\phi)$ that is similar to $\mathcal{T}$, and every side of $\mathcal{T}^{*}(\phi)$ touches $\mathcal{C}_{\phi}$ (see Fig. 3). The condition that $\mathcal{C}_{\phi}$ is entirely contained in $\mathcal{T}$ is equivalent to the fact that $\mathcal{T}^{*}(\phi)$ is smaller than $\mathcal{T}$. Hence the length of side $c$ of $\mathcal{T}$ is greater than the length of the respective side $c^{*}(\phi)$ of $\mathcal{T}^{*}(\phi)$. Using Fig. 3, we find

$$
c^{*}(\phi)=s(\phi) \csc \alpha+s(\phi+\alpha+\beta) \csc \beta+s(\phi+\alpha+\pi)(\cot \alpha+\cot \beta) .
$$

So we have

$$
\max _{0 \leq \phi<2 \pi} c^{*}(\phi) \leq c
$$

(The equals sign does not influence the calculation of the probabilities.) The radius $\rho$ of the incircle of $\mathcal{T}$ is given by $\rho=Q /(a+b+c)$ [5, p. 6]. Clearly, if $\max _{0 \leq \phi<\pi} w(\phi) \leq 2 \rho$ (cf. [4, p. 16]), then condition (1) always holds true. 

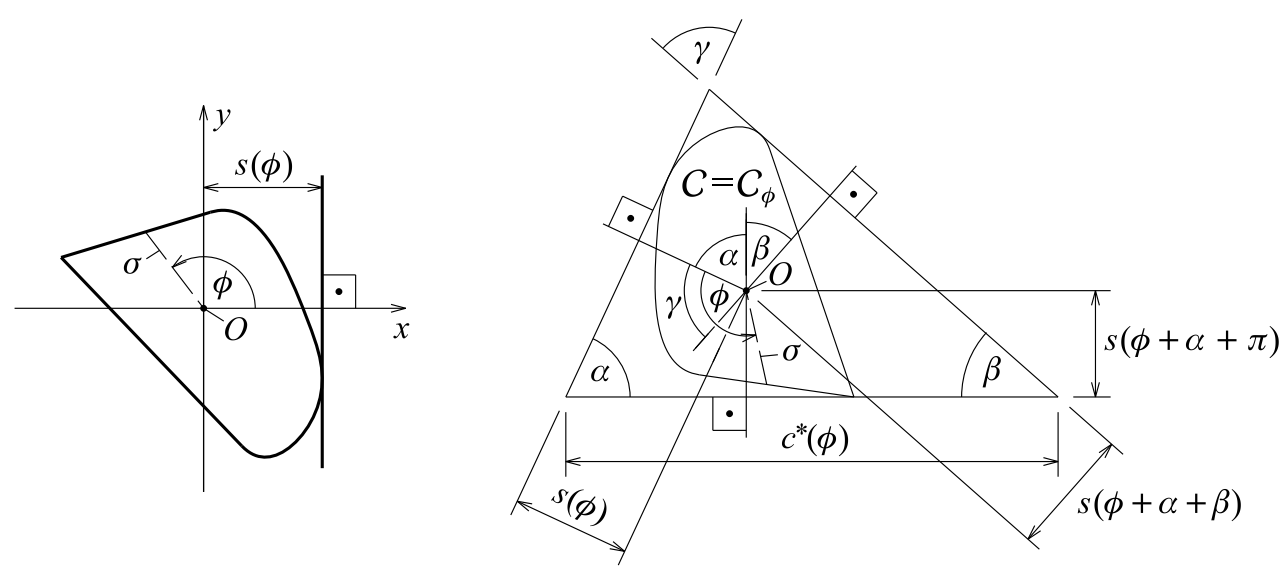

Fig. 2: Support function $s$

Fig. 3: Triangle $\mathcal{T}^{*}(\phi)$

Theorem 2.1. If condition (1) holds true, the probabilities $p(i)$ that $\mathcal{C}$ hits exactly $i$ triangles of $\mathcal{R}_{a, b, c}$ are given by

$$
\begin{aligned}
& p(1)=1-\frac{(a+b+c) u}{\pi Q}+\frac{\left(a^{2}+b^{2}+c^{2}\right) J(0)}{2 \pi Q^{2}}+\frac{b c f_{1}(\alpha)+c a f_{1}(\beta)+a b f_{1}(\gamma)}{\pi Q^{2}}, \\
& p(2)=\frac{(a+b+c) u}{\pi Q}-\frac{3\left(a^{2}+b^{2}+c^{2}\right) J(0)}{2 \pi Q^{2}}-\frac{b c f_{2}(\alpha)+c a f_{2}(\beta)+a b f_{2}(\gamma)}{\pi Q^{2}}, \\
& p(3)=\frac{3\left(a^{2}+b^{2}+c^{2}\right) J(0)}{2 \pi Q^{2}}+\frac{b c f_{3}(\alpha)+c a f_{3}(\beta)+a b f_{3}(\gamma)}{\pi Q^{2}}, \\
& p(4)=\frac{b c J(\alpha)+c a J(\beta)+a b J(\gamma)}{\pi Q^{2}}-\frac{\left(a^{2}+b^{2}+c^{2}\right) J(0)}{2 \pi Q^{2}}-\frac{F}{Q}, \\
& p(5)=0, \quad p(6)=\frac{F}{Q},
\end{aligned}
$$

with

$$
\begin{aligned}
& f_{1}(x)=I(x)-J(x), \quad f_{2}(x)=2 I(x)-3 J(x), \quad f_{3}(x)=I(x)-3 J(x), \\
& I(x)=\int_{0}^{\pi} w(\phi) w(\phi+x) \mathrm{d} \phi, \quad J(x)=\int_{0}^{2 \pi} s(\phi) s(\phi+x) \mathrm{d} \phi,
\end{aligned}
$$

and the expectation for the random number $Z$ of hit triangles by

$$
\mathrm{E}(Z)=1+\frac{(a+b+c) u}{\pi Q}+\frac{2 F}{Q} .
$$

Proof. It is sufficient to consider only the cases where $O \in \mathcal{Q}$. (It would even be sufficient to consider only the cases where $O \in \mathcal{T}$. But considering $O \in \mathcal{Q}$ is much more convenient.) 


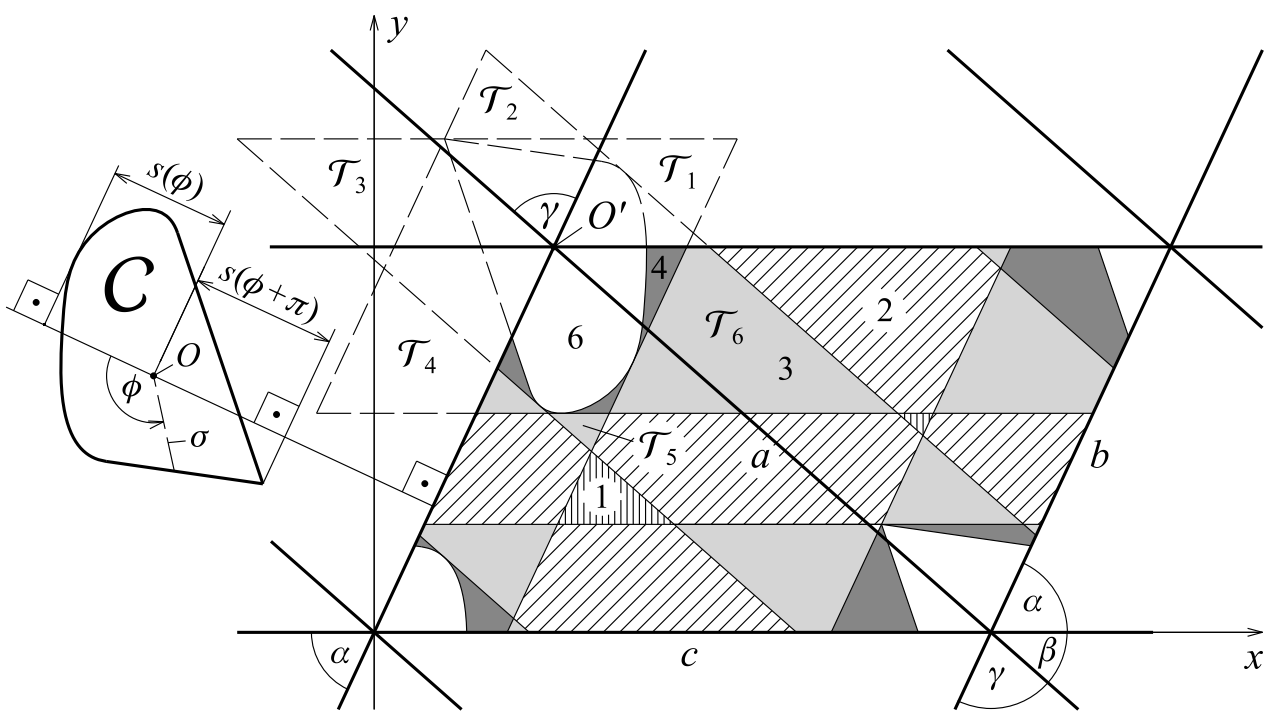

Fig. 4: Situation in $\mathcal{Q}$ for fixed value of the angle $\phi$

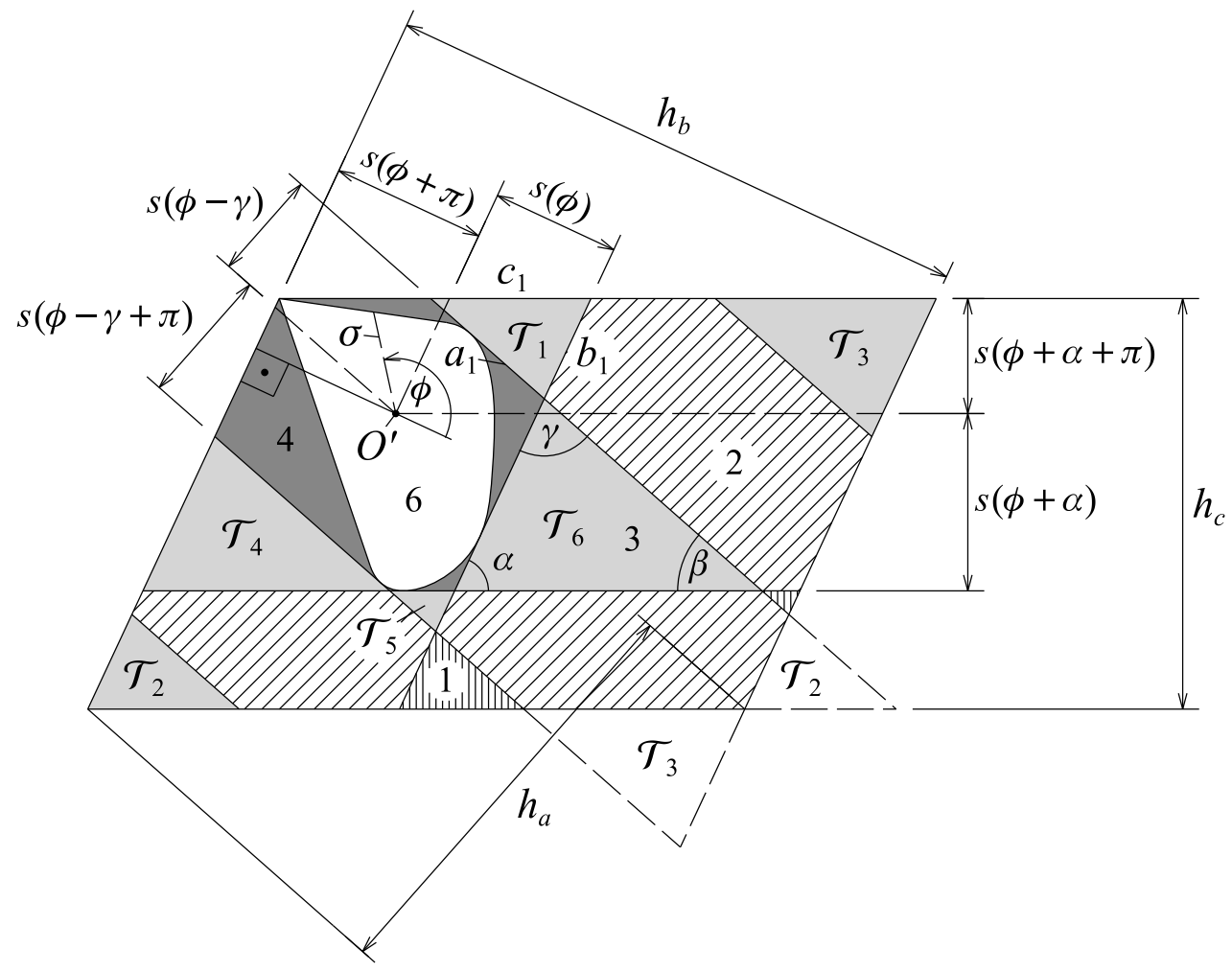

Fig. 5: Rearrangement of $\mathcal{Q}$ 
Since $\mathcal{C}$ is convex, it follows that $p(5)=0$. For fixed value of $\phi, \mathcal{C}$ hits exactly $i \in\{1,2,3,4,6\}$ parallelograms of $\mathcal{R}_{a, b, c}$ if $O$ is inside the set with number $i$ (see Fig. 4), where every set $i$ is the union of all sets with equal shading. In the following, we use the law of total probability in the form

$$
p(i)=\int_{0}^{2 \pi} p(i \mid \phi) \frac{\mathrm{d} \phi}{2 \pi},
$$

where $p(i \mid \phi)$ denotes the conditional probability of exactly $i$ hits for fixed value of $\phi$. We have

$$
p(i \mid \phi)=\frac{F_{i}(\phi)}{Q}
$$

where $F_{i}(\phi)$ is the area of set $i$. By rearranging the parallelogram $\mathcal{Q}$, one gets the parallelogram shown in Fig. 5. With the help of this figure it is easier to find and calculate the areas $F_{i}(\phi)$.

For every $\phi, F_{6}(\phi)$ is equal to $F$ since set 6 is a congruent copy of $\mathcal{C}$ (see Fig. 4 and 5), hence

$$
p(6)=\frac{1}{2 \pi Q} \int_{0}^{2 \pi} F \mathrm{~d} \phi=\frac{F}{2 \pi Q} \int_{0}^{2 \pi} \mathrm{d} \phi=\frac{F}{Q}
$$

(cf. [8, p. 321], [9, pp. 73-74]). Now, we determine the probability $p(3) . \mathcal{C}$, with direction $\phi$, hits exactly three triangles of $\mathcal{R}_{a, b, c}$ if $C \in \mathcal{T}_{1}(\phi) \cup \mathcal{T}_{2}(\phi) \cup \ldots$ $\cup \mathcal{T}_{6}(\phi)$. Every triangle $\mathcal{T}_{i}(\phi)$ is similar to the triangle $\mathcal{T}$. We denote by $a_{i}(\phi), b_{i}(\phi), c_{i}(\phi)$ the sides of $\mathcal{T}_{i}(\phi)$ with opposite angles $\alpha, \beta, \gamma$, respectively. Because of the said similarity, the area of $\mathcal{T}_{i}(\phi)$ is given by

$$
\frac{a_{i}^{2}(\phi)}{a^{2}} \frac{Q}{2}=\frac{b_{i}^{2}(\phi)}{b^{2}} \frac{Q}{2}=\frac{c_{i}^{2}(\phi)}{c^{2}} \frac{Q}{2}
$$

and, therefore,

$$
F_{3}(\phi)=\frac{Q}{2}\left(\frac{a_{1}^{2}(\phi)}{a^{2}}+\frac{c_{2}^{2}(\phi)}{c^{2}}+\frac{b_{3}^{2}(\phi)}{b^{2}}+\frac{a_{4}^{2}(\phi)}{a^{2}}+\frac{c_{5}^{2}(\phi)}{c^{2}}+\frac{b_{6}^{2}(\phi)}{b^{2}}\right),
$$

hence

$$
\begin{aligned}
p(3)=\frac{1}{4 \pi} & \left(\frac{1}{a^{2}} \int_{0}^{2 \pi} a_{1}^{2}(\phi) \mathrm{d} \phi+\frac{1}{c^{2}} \int_{0}^{2 \pi} c_{2}^{2}(\phi) \mathrm{d} \phi+\frac{1}{b^{2}} \int_{0}^{2 \pi} b_{3}^{2}(\phi) \mathrm{d} \phi\right. \\
& \left.+\frac{1}{a^{2}} \int_{0}^{2 \pi} a_{4}^{2}(\phi) \mathrm{d} \phi+\frac{1}{c^{2}} \int_{0}^{2 \pi} c_{5}^{2}(\phi) \mathrm{d} \phi+\frac{1}{b^{2}} \int_{0}^{2 \pi} b_{6}^{2}(\phi) \mathrm{d} \phi\right) .
\end{aligned}
$$

Using the support function $s$ of $\mathcal{C}$, one finds

$$
a_{1}(\phi)=s(\phi-\beta-\gamma) \csc \beta+s(\phi) \csc \gamma-s(\phi-\gamma)(\cot \beta+\cot \gamma) .
$$


Hitting probabilities for random convex bodies and lattices of triangles

We put $d(\beta, \gamma, \phi):=a_{1}(\phi)$. Due to the symmetry of $\mathcal{R}_{a, b, c}$,

$$
\begin{array}{ll}
c_{2}(\phi)=d(\alpha, \beta, \phi-\gamma), & b_{3}(\phi)=d(\gamma, \alpha, \phi-\gamma-\beta), \\
a_{4}(\phi)=d(\beta, \gamma, \phi+\alpha+\beta+\gamma), & c_{5}(\phi)=d(\alpha, \beta, \phi+\alpha+\beta), \\
b_{6}(\phi)=d(\gamma, \alpha, \phi+\alpha) . &
\end{array}
$$

Since $d(x, y, \cdot)$ with $(x, y) \in\{(\alpha, \beta),(\beta, \gamma),(\gamma, \alpha)\}$ is a $2 \pi$-periodic function, we get

$$
\begin{aligned}
\int_{0}^{2 \pi} a_{1}^{2}(\phi) \mathrm{d} \phi & =\int_{0}^{2 \pi} d^{2}(\beta, \gamma, \phi) \mathrm{d} \phi=\int_{0}^{2 \pi} a_{4}^{2}(\phi) \mathrm{d} \phi \\
\int_{0}^{2 \pi} b_{3}^{2}(\phi) \mathrm{d} \phi & =\int_{0}^{2 \pi} d^{2}(\gamma, \alpha, \phi) \mathrm{d} \phi=\int_{0}^{2 \pi} b_{6}^{2}(\phi) \mathrm{d} \phi \\
\int_{0}^{2 \pi} c_{2}^{2}(\phi) \mathrm{d} \phi & =\int_{0}^{2 \pi} d^{2}(\alpha, \beta, \phi) \mathrm{d} \phi=\int_{0}^{2 \pi} c_{5}^{2}(\phi) \mathrm{d} \phi
\end{aligned}
$$

hence

$$
p(3)=\frac{1}{2 \pi}\left(\frac{H(\beta, \gamma)}{a^{2}}+\frac{H(\gamma, \alpha)}{b^{2}}+\frac{H(\alpha, \beta)}{c^{2}}\right)
$$

where

$$
H(x, y):=\int_{0}^{2 \pi} d^{2}(x, y, \phi) \mathrm{d} \phi
$$

With

$$
\begin{aligned}
& d^{2}(\beta, \gamma, \phi+\beta+\gamma) \\
& =s^{2}(\phi) \csc ^{2} \beta+s^{2}(\phi+\beta+\gamma) \csc ^{2} \gamma+s^{2}(\phi+\beta)(\cot \beta+\cot \gamma)^{2} \\
& \quad+2 s(\phi) s(\phi+\beta+\gamma) \csc \beta \csc \gamma-2 s(\phi) s(\phi+\beta) \csc \beta(\cot \beta+\cot \gamma) \\
& \quad-2 s(\phi+\beta+\gamma) s(\phi+\beta) \csc \gamma(\cot \beta+\cot \gamma),
\end{aligned}
$$

and, using the the relations

$$
\begin{gathered}
\int_{0}^{2 \pi} s^{2}(\phi+\beta) \mathrm{d} \phi=\int_{0}^{2 \pi} s^{2}(\phi+\beta+\gamma) \mathrm{d} \phi=\int_{0}^{2 \pi} s^{2}(\phi) \mathrm{d} \phi \\
\int_{0}^{2 \pi} s(\phi+\beta) s(\phi+\beta+\gamma) \mathrm{d} \phi=\int_{0}^{2 \pi} s(\phi) s(\phi+\gamma) \mathrm{d} \phi \\
\int_{0}^{2 \pi} s(\phi) s(\phi+\beta+\gamma) \mathrm{d} \phi=\int_{0}^{2 \pi} s(\phi+\alpha) s(\phi+\alpha+\beta+\gamma) \mathrm{d} \phi \\
=\int_{0}^{2 \pi} s(\phi+\pi) s(\phi+\alpha) \mathrm{d} \phi,
\end{gathered}
$$


that result from the $2 \pi$-periodicity of $s$, one finds

$$
\begin{aligned}
H(\beta, \gamma)= & \int_{0}^{2 \pi} d^{2}(\beta, \gamma, \phi) \mathrm{d} \phi=\int_{0}^{2 \pi} d^{2}(\beta, \gamma, \phi+\beta+\gamma) \mathrm{d} \phi \\
= & {\left[\csc ^{2} \beta+\csc ^{2} \gamma+(\cot \beta+\cot \gamma)^{2}\right] \int_{0}^{2 \pi} s^{2}(\phi) \mathrm{d} \phi } \\
& +2 \csc \beta \csc \gamma \int_{0}^{2 \pi} s(\phi+\pi) s(\phi+\alpha) \mathrm{d} \phi \\
& -2(\cot \beta+\cot \gamma) \csc \beta \int_{0}^{2 \pi} s(\phi) s(\phi+\beta) \mathrm{d} \phi \\
& -2(\cot \beta+\cot \gamma) \csc \gamma \int_{0}^{2 \pi} s(\phi) s(\phi+\gamma) \mathrm{d} \phi
\end{aligned}
$$

From

$$
\begin{aligned}
& \int_{0}^{2 \pi} w(\phi) w(\phi+\alpha) \mathrm{d} \phi \\
& =\int_{0}^{2 \pi}[s(\phi)+s(\phi+\pi)][s(\phi+\alpha)+s(\phi+\alpha+\pi)] \mathrm{d} \phi \\
& =\int_{0}^{2 \pi}[s(\phi) s(\phi+\alpha)+s(\phi) s(\phi+\alpha+\pi)+s(\phi+\pi) s(\phi+\alpha) \\
& \quad+s(\phi+\pi) s(\phi+\alpha+\pi)] \mathrm{d} \phi \\
& =2 \int_{0}^{2 \pi} s(\phi) s(\phi+\alpha) \mathrm{d} \phi+2 \int_{0}^{2 \pi} s(\phi+\pi) s(\phi+\alpha) \mathrm{d} \phi
\end{aligned}
$$

we get, with the $\pi$-periodicity of $w$,

$$
\begin{aligned}
& \int_{0}^{2 \pi} s(\phi+\pi) s(\phi+\alpha) \mathrm{d} \phi \\
& =\frac{1}{2} \int_{0}^{2 \pi} w(\phi) w(\phi+\alpha) \mathrm{d} \phi-\int_{0}^{2 \pi} s(\phi) s(\phi+\alpha) \mathrm{d} \phi \\
& =\int_{0}^{\pi} w(\phi) w(\phi+\alpha) \mathrm{d} \phi-\int_{0}^{2 \pi} s(\phi) s(\phi+\alpha) \mathrm{d} \phi .
\end{aligned}
$$

Therefore, with the abbreviations

$$
I(x):=\int_{0}^{\pi} w(\phi) w(\phi+x) \mathrm{d} \phi \quad \text { and } \quad J(x):=\int_{0}^{2 \pi} s(\phi) s(\phi+x) \mathrm{d} \phi
$$


we find

$$
\begin{aligned}
\frac{H(\beta, \gamma)}{a^{2}}= & \frac{1}{a^{2}} \int_{0}^{2 \pi} d^{2}(\beta, \gamma, \phi) \mathrm{d} \phi=\frac{1}{a^{2}} \int_{0}^{2 \pi} d^{2}(\beta, \gamma, \phi+\beta+\gamma) \mathrm{d} \phi \\
= & {\left[\csc ^{2} \beta+\csc ^{2} \gamma+(\cot \beta+\cot \gamma)^{2}\right] J(0) } \\
& +2 \csc \beta \csc \gamma[I(\alpha)-J(\alpha)]-2(\cot \beta+\cot \gamma) \csc \beta J(\beta) \\
& -2(\cot \beta+\cot \gamma) \csc \gamma J(\gamma) \\
= & {\left[\frac{c^{2}}{(a c \sin \beta)^{2}}+\frac{b^{2}}{(a b \sin \gamma)^{2}}+\left(\frac{c \cos \beta}{a c \sin \beta}+\frac{b \cos \gamma}{a b \sin \gamma}\right)^{2}\right] J(0) } \\
& +\frac{2 b c}{a c \sin \beta a b \sin \gamma} I(\alpha)-\frac{2 b c}{a c \sin \beta a b \sin \gamma} J(\alpha) \\
& -2\left(\frac{c \cos \beta}{a c \sin \beta}+\frac{b \cos \gamma}{a b \sin \gamma}\right) \frac{c}{a c \sin \beta} J(\beta) \\
& -2\left(\frac{c \cos \beta}{a c \sin \beta}+\frac{b \cos \gamma}{a b \sin \gamma}\right) \frac{b}{a b \sin \gamma} J(\gamma) \\
= & \frac{\left(a^{2}+b^{2}+c^{2}\right) J(0)}{Q^{2}}+\frac{2 b c I(\alpha)}{Q^{2}}-\frac{2 b c J(\alpha)}{Q^{2}}-\frac{2 a c J(\beta)}{Q^{2}}-\frac{2 a b J(\gamma)}{Q^{2}}
\end{aligned}
$$

It follows that

$$
\begin{aligned}
p(3)= & \frac{1}{2 \pi Q^{2}}\left[\left(a^{2}+b^{2}+c^{2}\right) J(0)+2 b c I(\alpha)-2 b c J(\alpha)-2 c a J(\beta)-2 a b J(\gamma)\right. \\
& +\left(b^{2}+c^{2}+a^{2}\right) J(0)+2 c a I(\beta)-2 c a J(\beta)-2 a b J(\gamma)-2 b c J(\alpha) \\
& \left.+\left(c^{2}+a^{2}+b^{2}\right) J(0)+2 a b I(\gamma)-2 a b J(\gamma)-2 b c J(\alpha)-2 c a J(\beta)\right] \\
= & \frac{3\left(a^{2}+b^{2}+c^{2}\right) J(0)}{2 \pi Q^{2}}+\frac{b c I(\alpha)+c a I(\beta)+a b I(\gamma)}{\pi Q^{2}} \\
& -\frac{3[b c J(\alpha)+c a J(\beta)+a b J(\gamma)]}{\pi Q^{2}} .
\end{aligned}
$$

As the next step, we determine $p(4)$. Using Fig. 5, one sees that

$$
\begin{aligned}
F_{4}(\phi) & =\frac{w(\phi) w(\phi+\alpha)}{\sin \alpha}-\frac{a_{1}^{2}(\phi)}{a^{2}} \frac{Q}{2}-\frac{a_{4}^{2}(\phi)}{a^{2}} \frac{Q}{2}-F \\
& =\frac{b c w(\phi) w(\phi+\alpha)}{Q}-\frac{a_{1}^{2}(\phi)}{a^{2}} \frac{Q}{2}-\frac{a_{4}^{2}(\phi)}{a^{2}} \frac{Q}{2}-F .
\end{aligned}
$$

So we find

$$
\begin{aligned}
p(4)= & \frac{1}{2 \pi Q}\left[\frac{b c}{Q} \int_{0}^{2 \pi} w(\phi) w(\phi+\alpha) \mathrm{d} \phi-\frac{Q}{2 a^{2}}\left(\int_{0}^{2 \pi} a_{1}^{2}(\phi) \mathrm{d} \phi\right.\right. \\
& \left.\left.+\int_{0}^{2 \pi} a_{4}^{2}(\phi) \mathrm{d} \phi\right)-F \int_{0}^{2 \pi} \mathrm{d} \phi\right]
\end{aligned}
$$




$$
\begin{aligned}
& =\frac{1}{2 \pi Q}\left[\frac{2 b c I(\alpha)}{Q}-\frac{Q}{a^{2}} \int_{0}^{2 \pi} d^{2}(\beta, \gamma, \phi) \mathrm{d} \phi-2 \pi F\right] \\
& =\frac{b c I(\alpha)}{\pi Q^{2}}-\frac{H(\beta, \gamma)}{2 \pi a^{2}}-\frac{F}{Q} .
\end{aligned}
$$

Taking into account the symmetry of $\mathcal{R}_{a, b, c}$, we also have

$$
\begin{aligned}
p(4)= & \frac{1}{3}\left[\left(\frac{b c I(\alpha)}{\pi Q^{2}}-\frac{H(\beta, \gamma)}{2 \pi a^{2}}-\frac{F}{Q}\right)+\left(\frac{c a I(\beta)}{\pi Q^{2}}-\frac{H(\gamma, \alpha)}{2 \pi b^{2}}-\frac{F}{Q}\right)\right. \\
& \left.+\left(\frac{a b I(\gamma)}{\pi Q^{2}}-\frac{H(\alpha, \beta)}{2 \pi c^{2}}-\frac{F}{Q}\right)\right] \\
= & \frac{b c I(\alpha)+c a I(\beta)+a b I(\gamma)}{3 \pi Q^{2}}-\frac{1}{3} p(3)-\frac{F}{Q} \\
= & \frac{b c I(\alpha)+c a I(\beta)+a b I(\gamma)}{3 \pi Q^{2}}-\frac{1}{3}\left(\frac{3\left(a^{2}+b^{2}+c^{2}\right) J(0)}{2 \pi Q^{2}}\right. \\
& \left.+\frac{b c I(\alpha)+c a I(\beta)+a b I(\gamma)}{\pi Q^{2}}-\frac{3[b c J(\alpha)+c a J(\beta)+a b J(\gamma)]}{\pi Q^{2}}\right)-\frac{F}{Q} \\
= & \frac{b c J(\alpha)+c a J(\beta)+a b J(\gamma)}{\pi Q^{2}}-\frac{\left(a^{2}+b^{2}+c^{2}\right) J(0)}{2 \pi Q^{2}}-\frac{F}{Q} .
\end{aligned}
$$

The next probability to determine is $p(1)$. One finds

$$
\begin{aligned}
F_{1}(\phi)= & \frac{\left[h_{b}-w(\phi)\right]\left[h_{c}-w(\phi+\alpha)\right]}{\sin \alpha}-\left(\frac{\left[h_{c}-w(\phi+\alpha)\right] w(\phi-\gamma)}{\sin \beta}\right. \\
& \left.-\frac{c_{2}^{2}(\phi)}{c^{2}} \frac{Q}{2}-\frac{c_{5}^{2}(\phi)}{c^{2}} \frac{Q}{2}\right) \\
= & \frac{h_{b} h_{c}}{\sin \alpha}-\frac{h_{b} w(\phi+\alpha)}{\sin \alpha}-\frac{h_{c} w(\phi)}{\sin \alpha}+\frac{w(\phi) w(\phi+\alpha)}{\sin \alpha}-\frac{h_{c} w(\phi-\gamma)}{\sin \beta} \\
& +\frac{w(\phi+\alpha) w(\phi-\gamma)}{\sin \beta}+\frac{Q}{2 c^{2}}\left[d^{2}(\alpha, \beta, \phi-\gamma)+d^{2}(\alpha, \beta, \phi+\alpha+\beta)\right] .
\end{aligned}
$$

Using $h_{b}=a \sin \gamma=c \sin \alpha, h_{c}=b \sin \alpha=a \sin \beta, w(\phi-\gamma)=w(\phi+\alpha+\beta)$, and $Q=a b \sin \gamma$ we get

$$
\begin{aligned}
F_{1}(\phi) & =Q-c w(\phi+\alpha)-b w(\phi)+\frac{b c}{Q} w(\phi) w(\phi+\alpha)-a w(\phi+\alpha+\beta) \\
& +\frac{a c}{Q} w(\phi+\alpha) w(\phi+\alpha+\beta)+\frac{Q}{2 c^{2}}\left[d^{2}(\alpha, \beta, \phi-\gamma)+d^{2}(\alpha, \beta, \phi+\alpha+\beta)\right] .
\end{aligned}
$$

This, with

$$
\int_{0}^{\pi} w(\phi) \mathrm{d} \phi=\int_{0}^{\pi}[s(\phi)+s(\phi+\pi)] \mathrm{d} \phi=\int_{0}^{2 \pi} s(\phi) \mathrm{d} \phi=u
$$


(see $[11$, p. 3]), and

$$
\begin{aligned}
\int_{0}^{2 \pi} w(\phi+\alpha) w(\phi+\alpha+\beta) \mathrm{d} \phi & =2 \int_{0}^{\pi} w(\phi+\alpha) w(\phi+\alpha+\beta) \mathrm{d} \phi \\
& =2 \int_{0}^{\pi} w(\phi) w(\phi+\beta) \mathrm{d} \phi
\end{aligned}
$$

gives

$$
\begin{aligned}
p(1)= & \frac{1}{2 \pi Q}\left(Q \int_{0}^{2 \pi} \mathrm{d} \phi-2(a+b+c) \int_{0}^{\pi} w(\phi) \mathrm{d} \phi\right. \\
& +\frac{2 b c}{Q} \int_{0}^{\pi} w(\phi) w(\phi+\alpha) \mathrm{d} \phi+\frac{2 a c}{Q} \int_{0}^{\pi} w(\phi) w(\phi+\beta) \mathrm{d} \phi \\
& \left.+\frac{Q}{c^{2}} \int_{0}^{2 \pi} d^{2}(\alpha, \beta, \phi) \mathrm{d} \phi\right) \\
= & 1-\frac{(a+b+c) u}{\pi Q}+\frac{b c I(\alpha)}{\pi Q^{2}}+\frac{c a I(\beta)}{\pi Q^{2}}+\frac{H(\alpha, \beta)}{2 \pi c^{2}} .
\end{aligned}
$$

We now obtain, with the symmetry of $\mathcal{R}_{a, b, c}$,

$$
\begin{aligned}
p(1)= & \frac{1}{3}\left(1-\frac{(a+b+c) u}{\pi Q}+\frac{b c I(\alpha)}{\pi Q^{2}}+\frac{c a I(\beta)}{\pi Q^{2}}+\frac{H(\alpha, \beta)}{2 \pi c^{2}}\right. \\
& +1-\frac{(b+c+a) u}{\pi Q}+\frac{c a I(\beta)}{\pi Q^{2}}+\frac{a b I(\gamma)}{\pi Q^{2}}+\frac{H(\beta, \gamma)}{2 \pi a^{2}} \\
& \left.+1-\frac{(c+a+b) u}{\pi Q}+\frac{a b I(\gamma)}{\pi Q^{2}}+\frac{b c I(\alpha)}{\pi Q^{2}}+\frac{H(\gamma, \alpha)}{2 \pi b^{2}}\right) \\
= & 1-\frac{(a+b+c) u}{\pi Q}+\frac{2[b c I(\alpha)+c a I(\beta)+a b I(\gamma)]}{3 \pi Q^{2}}+\frac{1}{3} p(3) \\
= & 1-\frac{(a+b+c) u}{\pi Q}+\frac{\left(a^{2}+b^{2}+c^{2}\right) J(0)}{2 \pi Q^{2}}+\frac{b c I(\alpha)+c a I(\beta)+a b I(\gamma)}{\pi Q^{2}} \\
& -\frac{b c J(\alpha)+c a J(\beta)+a b J(\gamma)}{\pi Q^{2}} .
\end{aligned}
$$

Now we determine the expression for $p(2)$. Let $E_{2}$ denote the event that $\mathcal{C}$ hits exactly two triangles of $\mathcal{R}_{a, b, c}$; let further $E_{a}, E_{b}$, and $E_{c}$ denote the events that $\mathcal{C}$ hits side $a, b$, and $c$, respectively. Since the events $E_{2} \cap E_{a}, E_{2} \cap E_{b}$ and $E_{2} \cap E_{c}$ are pairwise disjoint,

$$
p(2)=P\left(E_{2}\right)=P\left(E_{2} \cap E_{a}\right)+P\left(E_{2} \cap E_{b}\right)+P\left(E_{2} \cap E_{c}\right) .
$$

We have

$$
P\left(E_{2} \cap E_{a}\right)=\int_{0}^{2 \pi} P\left(E_{2} \cap E_{a} \mid \phi\right) \frac{\mathrm{d} \phi}{2 \pi}, \quad P\left(E_{2} \cap E_{a} \mid \phi\right)=\frac{F_{2, a}(\phi)}{Q} .
$$


For the area $F_{2, a}(\phi)$, we can write

$$
\begin{aligned}
F_{2, a}(\phi)= & \frac{w(\phi-\gamma)\left[h_{b}-w(\phi)\right]}{\sin \gamma}-\frac{b_{3}^{2}(\phi)}{b^{2}} \frac{Q}{2}-\frac{b_{6}^{2}(\phi)}{b^{2}} \frac{Q}{2} \\
= & \frac{h_{b} w(\phi-\gamma)}{\sin \gamma}-\frac{w(\phi-\gamma) w(\phi)}{\sin \gamma}-\frac{Q}{2 b^{2}}\left[d^{2}(\gamma, \alpha, \phi-\gamma-\beta)\right. \\
& \left.+d^{2}(\gamma, \alpha, \phi+\alpha)\right] \\
= & a w(\phi-\gamma)-\frac{a b}{Q} w(\phi-\gamma) w(\phi)-\frac{Q}{2 b^{2}}\left[d^{2}(\gamma, \alpha, \phi-\gamma-\beta)\right. \\
& \left.+d^{2}(\gamma, \alpha, \phi+\alpha)\right] .
\end{aligned}
$$

Hence, with $\int_{0}^{2 \pi} w(\phi-\gamma) w(\phi) \mathrm{d} \phi=2 \int_{0}^{\pi} w(\phi) w(\phi+\gamma) \mathrm{d} \phi$,

$$
\begin{aligned}
P\left(E_{2} \cap E_{a}\right)= & \frac{1}{2 \pi Q}\left[2 a \int_{0}^{\pi} w(\phi) \mathrm{d} \phi-\frac{2 a b}{Q} \int_{0}^{\pi} w(\phi) w(\phi+\gamma) \mathrm{d} \phi\right. \\
& \left.-\frac{Q}{b^{2}} \int_{0}^{2 \pi} d^{2}(\gamma, \alpha, \phi) \mathrm{d} \phi\right]=\frac{a u}{\pi Q}-\frac{a b I(\gamma)}{\pi Q^{2}}-\frac{H(\gamma, \alpha)}{2 \pi b^{2}} .
\end{aligned}
$$

Due to the symmetry of $\mathcal{R}_{a, b, c}$, we also have

$$
\begin{aligned}
& P\left(E_{2} \cap E_{b}\right)=\frac{b u}{\pi Q}-\frac{b c I(\alpha)}{\pi Q^{2}}-\frac{H(\alpha, \beta)}{2 \pi c^{2}}, \\
& P\left(E_{2} \cap E_{c}\right)=\frac{c u}{\pi Q}-\frac{c a I(\beta)}{\pi Q^{2}}-\frac{H(\beta, \gamma)}{2 \pi a^{2}} .
\end{aligned}
$$

It follows that

$$
\begin{aligned}
p(2)= & \frac{(a+b+c) u}{\pi Q}-\frac{b c I(\alpha)+c a I(\beta)+a b I(\gamma)}{\pi Q^{2}}-p(3) \\
= & \frac{(a+b+c) u}{\pi Q}-\frac{3\left(a^{2}+b^{2}+c^{2}\right) J(0)}{2 \pi Q^{2}}-\frac{2[b c I(\alpha)+c a I(\beta)+a b I(\gamma)]}{\pi Q^{2}} \\
& +\frac{3[b c J(\alpha)+c a J(\beta)+a b J(\gamma)]}{\pi Q^{2}} .
\end{aligned}
$$

Finally, for the expectation of the random number $Z$ of hit triangles one easily finds

$$
\mathrm{E}(Z)=\sum_{i=1}^{6} i p(i)=1+\frac{(a+b+c) u}{\pi Q}+\frac{2 F}{Q} .
$$




\section{References}

[1] U. Bäsel, A. Duma, Intersection probabilities for random convex bodies and lattices of parallelograms, Fernuniversität Hagen: Seminarberichte aus der Fakultät für Mathematik und Informatik 86 (2014), 1-14.

[2] U. Bäsel, A. Duma, Intersection probabilities for random convex bodies and lattices of parallelograms (II), Fernuniversität Hagen: Seminarberichte aus der Fakultät für Mathematik und Informatik 86 (2014), 101108.

[3] R. Böttcher, Das Buffonsche Experiment im Dreieckgitter, Fernuniversität Hagen, Fachbereich Mathematik, 2002, 16 pages, unpublished.

[4] A. Duma, M. Stoka, Geometrical probabilities for convex test bodies, Beitr. Algebra Geom. 40 No. 1 (1999), 15-25.

[5] W. Göhler, Höhere Mathematik - Formeln und Hinweise, Deutscher Verlag für Grundstoffindustrie, 9. Aufl., Leipzig, 1985.

[6] A. A. Markoff, Wahrscheinlichkeitsrechnung, B. G. Teubner, Leipzig, Berlin, 1912.

[7] M. Pettineo, A Buffon type problem for triangular lattices, Rend. Circ. Mat. Palermo, Serie II, Suppl. 50 (1997), 289-298.

[8] D. Ren, G. Zhang, Random convex sets in a lattice of parallelograms, Acta Math. Sci. 11 (1991), 317-326.

[9] D. Ren, Topics in Integral Geometry, World Scientific, Singapore, New Jersey, London, Hong Kong, 1994.

[10] L. A. Santaló, Sur quelques problèmes de probabilités géométriques, Tôhoku Math. J. 47 (1940), 159-171.

[11] L. A. Santaló, Integral Geometry and Geometric Probability, AddisonWesley, London, 1976.

Received: September 1, 2014; Published: November 25, 2014 